\title{
Variants of Equivariant Seiberg-Witten Floer Homology
}

\author{
Matilde Marcolli, Bai-Ling Wang
}

\begin{abstract}
For a rational homology 3 -sphere $Y$ with a $\operatorname{Spin}^{c}$ structure $\mathfrak{s}$, we show that simple algebraic manipulations of our construction of equivariant Seiberg-Witten Floer homology in [5] lead to a collection of variants $H F_{*, U(1)}^{S W,-}(Y, \mathfrak{s}), H F_{*, U(1)}^{S W, \infty}(Y, \mathfrak{s}) H F_{*, U(1)}^{S W,+}(Y, \mathfrak{s}), \widehat{H F}_{*}^{S W}(Y, \mathfrak{s})$ and $H F_{r e d, *}^{S W}(Y, \mathfrak{s})$ which are topological invariants. We establish a long exact sequence relating $H F_{*, U(1)}^{S W, \pm}(Y, \mathfrak{s})$ and $H F_{*, U(1)}^{S W, \infty}(Y, \mathfrak{s})$. We show they satisfy a duality under orientation reversal, and we explain their relation to the equivariant Seiberg-Witten Floer (co)homologies introduced in [5]. We conjecture the equivalence of these versions of equivariant Seiberg-Witten Floer homology with the Heegaard Floer invariants introduced by Ozsváth and Szabó.
\end{abstract}

Key words: rational homology 3-spheres, equivariant Seiberg-Witten Floer homology, $\operatorname{Spin}^{c}$ structures, topological invariants.

Mathematics Subject Classification. Primary: 57R58. Secondary: 57R57, 58J10.

\section{Introduction}

For any rational homology 3 -sphere $Y$ with a $\operatorname{Spin}^{c}$ structure $\mathfrak{s}$, we constructed in [5] an equivariant Seiberg-Witten Floer homology $\operatorname{HF}_{* U(1)}^{S W}(Y, \mathfrak{s})$, which is a topological invariant. In this paper, we will generalize this construction to provide a collection of equivariant Seiberg-Witten Floer homologies $H F_{*, U(1)}^{S W,-}(Y, \mathfrak{s}), H F_{*, U(1)}^{S W, \infty}(Y, \mathfrak{s}), H F_{*, U(1)}^{S W,+}(Y, \mathfrak{s}), \widehat{H F}_{*}^{S W}(Y, \mathfrak{s})$ and $H F_{r e d, *}^{S W}(Y, \mathfrak{s})$, all of which are topological invariants, such that $H F_{*, U(1)}^{S W,+}(Y, \mathfrak{s})$ is isomorphic to the equivariant Seiberg-Witten Floer homology $H F_{*, U(1)}^{S W}(Y, \mathfrak{s})$ constructed in [5]. The construction utilizes the $U(1)$-invariant forms on $U(1)$-manifolds twisted with coefficients in the Laurent polynomial algebra over integers.

In analogy to Austin and Braam's construction of equivariant instanton Floer homology in [1], the equivariant Seiberg-Witten Floer homology $H F_{*, U(1)}^{S W}(Y, \mathfrak{s})$ is the homology of the complex 
$\left(C F_{*, U(1)}^{S W}(Y, \mathfrak{s}), D\right)$, where $C F_{*, U(1)}^{S W}(Y, \mathfrak{s})$ is generated by equivariant de Rham forms over all $U(1)$-orbits of the solutions of 3-dimensional Seiberg-Witten equations on $(Y, \mathfrak{s})$ modulo based gauge transformations (Cf.[5]). More specifically,

$$
C F_{*, U(1)}^{S W}(Y, \mathfrak{s})=\bigoplus_{a \in \mathcal{M}_{Y}^{*}(\mathfrak{s})} \mathbb{Z}[\Omega] \otimes\left(\mathbb{Z} \eta_{a} \oplus \mathbb{Z} 1_{a}\right) \oplus \mathbb{Z}[\Omega] \otimes \mathbb{Z} 1_{\theta}
$$

where $\mathcal{M}_{Y}(\mathfrak{s})=\mathcal{M}_{Y}^{*}(\mathfrak{s}) \cup\{\theta\}$ is the equivalence classes of solutions to the Seiberg-Witten equations for a good pair of metric and perturbations, consists of the irreducible monopoles $\mathcal{M}_{Y}^{*}(\mathfrak{s})$ and the unique reducible monopole $\theta$. We used the notation $\eta_{a}$ to denote a 1 -form on $O_{a} \cong S^{1}$, such that the cohomology class $\left[\eta_{a}\right]$ is an integral generator of $H^{1}\left(O_{a}\right)$. Similarly, we denote by $1_{a}$ the 0 -form given by the constant function.

Each generator is endowed with a grading such that, for any $k \geq 0$,

$$
\operatorname{gr}\left(\Omega^{k} \otimes \eta_{a}\right)=2 k+\operatorname{gr}(a), \operatorname{gr}\left(\Omega^{k} \otimes 1_{a}\right)=2 k+\operatorname{gr}(a)+1, \text { and } \operatorname{gr}\left(\Omega^{k} \otimes 1_{\theta}\right)=2 k,
$$

where $g r: \mathcal{M}_{Y}^{*}(\mathfrak{s}) \rightarrow \mathbb{Z}$ is the relative grading with respect to the reducible monopole $\theta$. This corresponds to grading equivariant de Rham forms on each orbit $O_{a}$ by codimension (Cf.[5] $\S 5$ for details).

The differential operator $D$ can be expressed explicitly in components as the form:

$$
\begin{aligned}
D\left(\Omega^{k} \otimes \eta_{a}\right)= & \sum_{\substack{b \in \mathcal{M}_{Y}^{*}(\mathfrak{s}) \\
g r(a)-g r(b)=1}} n_{a b} \Omega^{k} \otimes \eta_{b}+\sum_{\substack{c \in \mathcal{M}_{Y}^{*}(\mathfrak{s}) \\
g r(a)-g r(c)=2}} m_{a c} \Omega^{k} \otimes 1_{c}-\Omega^{k-1} \otimes 1_{a} \\
& +n_{a \theta} \Omega^{k} \otimes 1_{\theta}(\text { if gr }(\mathrm{a})=1) ; \\
D\left(\Omega^{k} \otimes 1_{a}\right)= & -\sum_{\substack{b \in \mathcal{M}_{Y}^{*}(\mathfrak{s}) \\
g r(a)-g r(b)=1}} n_{a b} \Omega^{k} \otimes 1_{b} ; \\
D\left(\Omega^{k} \otimes 1_{\theta}\right)= & \sum_{\substack{d \in \mathcal{M}_{Y}^{*}(\mathfrak{s}) \\
g r(d)=-2}} n_{\theta d} \Omega^{k} \otimes 1_{d} .
\end{aligned}
$$

where $n_{a b}, n_{a \theta}$ and $n_{\theta d}$ is the counting of flowlines from $a$ to $b$ (if $\operatorname{gr}(a)-\operatorname{gr}(b)=1$ ), from $a$ to $\theta$ (if $\operatorname{gr}(a)=1$ ) and from $\theta$ to $d$ (if $\operatorname{gr}(d)=-2$ ), and $m_{a c}$ (if $\operatorname{gr}(a)-\operatorname{gr}(c)=2$ ) is described as a relative Euler number associated to the 2-dimensional moduli space of flowlines from $a$ to $c$ (Cf. Lemma 5.7 of [5]). In the next section, we shall briefly review the construction and various relations among the coefficients, as established in [5]. These identities ensure that $D^{2}=0$. Notice that, in the complex $C F_{*, U(1)}^{S W}(Y, \mathfrak{s})$ and in the expression of the differential operator, only terms with non-negative powers of $\Omega$ are considered. We modify the construction as follows. 
Definition 1.1. Let $C F_{*, U(1)}^{S W, \infty}(Y, \mathfrak{s})$ be the graded complex generated by

$$
\left\{\Omega^{k} \otimes \eta_{a}, \Omega^{k} \otimes 1_{a}, \Omega^{k} \otimes 1_{\theta}: a \in \mathcal{M}_{Y}^{*}(\mathfrak{s}), k \in \mathbb{Z} \cdot\right\}
$$

with the grading gr and the differential operator D given by (2) and (3) respectively. Let $C F_{*, U(1)}^{S W,-}(Y, \mathfrak{s})$ be the subcomplex of $C F_{*, U(1)}^{S W, \infty}(Y, \mathfrak{s})$, generated by those generators with negative power of $\Omega$. The quotient complex is denoted by $C F_{*, U(1)}^{S W,+}(Y, \mathfrak{s})$. Their homologies are denoted by $H F_{*, U(1)}^{S W, \infty}(Y, \mathfrak{s}), H F_{*, U(1)}^{S W,-}(Y, \mathfrak{s})$ and $H F_{*, U(1)}^{S W,+}(Y, \mathfrak{s})$ respectively.

The main results in this paper relate these homologies to the equivariant Seiberg-Witten-Floer homology $H F_{*, U(1)}^{S W}(Y, \mathfrak{s})$ and cohomology $H F_{U(1)}^{S W, *}(Y, \mathfrak{s})$ constructed in [5] and establish some of their main properties.

Theorem 1.2. For any rational homology 3-sphere $Y$ with a $\operatorname{Spin}^{c}$ structure $\mathfrak{s} \in \operatorname{Spin}^{c}(Y)$, these homologies satisfy the following properties:

1. $H F_{*, U(1)}^{S W, \infty}(Y, \mathfrak{s}) \cong \mathbb{Z}\left[\Omega, \Omega^{-1}\right]$.

2. $H F_{*, U(1)}^{S W,+}(Y, \mathfrak{s}) \cong H F_{*, U(1)}^{S W}(Y, \mathfrak{s})$ where $H F_{*, U(1)}^{S W}(Y, \mathfrak{s})$ is the equivariant Seiberg-Witten Floer homology for $(Y, \mathfrak{s})$ constructed in [5].

3. $H F_{*, U(1)}^{S W,-}(Y, \mathfrak{s}) \cong H F_{U(1)}^{S W, *}(-Y, \mathfrak{s})$ where $H F_{U(1)}^{S W, *}(-Y, \mathfrak{s})$ is the equivariant Seiberg-Witten Floer cohomology for $(-Y, \mathfrak{s})$ constructed in [5].

4. There exists a long exact sequence

$$
\cdots \rightarrow H F_{*, U(1)}^{S W,-}(Y, \mathfrak{s}) \stackrel{l_{*}}{\longrightarrow} H F_{*, U(1)}^{S W, \infty}(Y, \mathfrak{s}) \stackrel{\pi_{*}}{\longrightarrow} H F_{*, U(1)}^{S W,+}(Y, \mathfrak{s}) \stackrel{\delta_{*}}{\longrightarrow} H F_{*-1, U(1)}^{S W,-}(Y, \mathfrak{s}) \rightarrow \cdots
$$

relating these homologies. Moreover, $\quad H F_{*, U(1)}^{S W,-}(Y, \mathfrak{s}), \quad H F_{*, U(1)}^{S W, \infty}(Y, \mathfrak{s}), \quad H F_{*, U(1)}^{S W,+}(Y, \mathfrak{s})$ and $H F_{r e d, *}^{S W}(Y, \mathfrak{s})=\operatorname{Coker}\left(\pi_{*}\right) \cong \operatorname{Ker}\left(l_{*-1}\right)$ are all topological invariants of $(Y, \mathfrak{s})$.

5. There is a u-action on $H F_{*, U(1)}^{S W,-}(Y, \mathfrak{s}), H F_{*, U(1)}^{S W, \infty}(Y, \mathfrak{s})$ and $H F_{*, U(1)}^{S W,+}(Y, \mathfrak{s})$ respectively which decreases the degree by two, and is related to the cutting down moduli spaces of flowlines by a geometric representative of a degree 2 characteristic form. The long exact sequence (4) is a long exact sequence of $\mathbb{Z}[u]$-modules. 
6. There is a homology group $\widehat{H F}_{*}^{S W}(Y, \mathfrak{s})$, which is also a topological invariant of $(Y, \mathfrak{s})$, such that the following sequence is exact:

$$
\cdots \rightarrow \widehat{H F}_{*}^{S W}(Y, \mathfrak{s}) \longrightarrow H F_{*, U(1)}^{S W,+}(Y, \mathfrak{s}) \stackrel{u}{\longrightarrow} H F_{*-2, U(1)}^{S W,+}(Y, \mathfrak{s}) \longrightarrow \widehat{H F}_{*-1}^{S W}(Y, \mathfrak{s}) \rightarrow \cdots
$$

and that $\widehat{H F}^{S W}(Y, \mathfrak{s})$ is non-trivial if and only if $\operatorname{HF}_{*, U(1)}^{S W,+}(Y, \mathfrak{s})$ is non-trivial.

The $u$-action in the main theorem is induced from a $u$-action on the chain complex

$$
u: \quad C F_{*, U(1)}^{S W, \infty} \rightarrow C F_{*, U(1)}^{S W, \infty}
$$

which decreases the degree by 2 . We will show that this $u$-action is homotopic to the obvious $\Omega^{-1}$ action on the chain complex $C F_{*, U(1)}^{S W, \infty}$. Thus, the induced $u$-action on $H F_{*, U(1)}^{S W, \pm}(Y, \mathfrak{s})$ endows them with $\mathbb{Z}[u]$-module structures.

Let $\widehat{C F}_{*}^{S W}(Y, \mathfrak{s})$ be the subcomplex of $C F_{*, U(1)}^{S W,+}(Y, \mathfrak{s})$ such that the following sequence is a short exact sequence of chain complexes:

$$
0 \rightarrow \widehat{C F}_{*}^{S W}(Y, \mathfrak{s}) \longrightarrow C F_{*, U(1)}^{S W,+}(Y, \mathfrak{s}) \stackrel{\Omega^{-1}}{\longrightarrow} C F_{*, U(1)}^{S W,+}(Y, \mathfrak{s}) \rightarrow 0
$$

We can define $\widehat{H F}_{*}^{S W}(Y, \mathfrak{s})$ to be the homology of $\widehat{C F}_{*}^{S W}(Y, \mathfrak{s})$.

In recent work [7] [8], Ozsváth and Szabó introduced Heegaard Floer invariants $H F_{*}^{ \pm}(Y, \mathfrak{s})$, $H F_{*}^{\infty}(Y, \mathfrak{s}), \widehat{H F}_{*}(Y, \mathfrak{s})$, and $H F_{r e d, *}(Y, \mathfrak{s})$, with exact sequences relating them. In view of their construction, the result of Theorem 1.2, together with the identification of our $\operatorname{HF}_{*, U(1)}^{S W, \infty}(Y, \mathfrak{s})$ and the $H F_{*}^{\infty}(Y, \mathfrak{s})$ of Ozsváth and Szabó, suggest the following conjecture.

Conjecture 1.3. For any rational homology 3-sphere $Y$ with a $\operatorname{Spin}^{c}$ structure $\mathfrak{s} \in \operatorname{Spin}^{c}(Y)$, there are isomorphisms

$$
\begin{aligned}
H F_{*, U(1)}^{S W,+}(Y, \mathfrak{s}) \cong H F_{*}^{+}(Y, \mathfrak{s}), & H F_{*, U(1)}^{S W,-}(Y, \mathfrak{s}) \cong H F_{*}^{-}(Y, \mathfrak{s}) ; \\
\widehat{H F}_{*}^{S W}(Y, \mathfrak{s}) \cong \widehat{H F}_{*}(Y, \mathfrak{s}), & H F_{r e d, *}^{S W}(Y, \mathfrak{s}) \cong H F_{r e d, *}(Y, \mathfrak{s}) .
\end{aligned}
$$

Acknowledgments This research was supported in part by the Humboldt Foundation's Sofja Kovalevskaya Award. 


\section{Review of equivariant Seiberg-Witten Floer homology}

In this section, we recall some of basic ingredients in the definition of the equivariant Seiebrg-Witten Floer homology from [5] (See [5] for all the details).

Let $(Y, \mathfrak{s})$ be a rational homology 3 -sphere $Y$ with a $\operatorname{Spin}^{c}$ structure $\mathfrak{s} \in \operatorname{Spin}^{c}(Y)$. For a good pair of metric and perturbation (a co-closed imaginary-valued 1-form $\nu$ ) on $Y$, the 3-dimensional Seiebrg-Witten equations on $(Y, \mathfrak{s})$ (Cf. [2] [3] [4] [5]):

$$
\left\{\begin{array}{l}
* F_{A}=\sigma(\psi, \psi)+\nu \\
\partial_{A} \psi=0
\end{array}\right.
$$

for a pair of $\operatorname{Spin}^{c}$ connection $A$ and a spinor $\psi$, have only finitely many irreducible solutions (modulo the gauge transformations), denoted by $\mathcal{M}_{Y}^{*}(\mathfrak{s})$ the set of equivalence classes of irreducible solutions to (6), and $\theta$ is the unique reducible solution (modulo the gauge transformations). Write $\mathcal{M}_{Y}(\mathfrak{s})=\mathcal{M}_{Y}^{*}(\mathfrak{s}) \cup\{\theta\}$.

Gauge classes of finite energy solutions to the 4-dimensional Seiebrg-Witten equations, perturbed as in [2] [3] [5], can be regarded as moduli spaces of flowlines of the Chern-Simons-Dirac functional on the gauge equivalence classes of $\operatorname{Spin}^{c}$ connections and spinors for $(Y, \mathfrak{s})$. These can be partitioned into moduli spaces of flowlines between pairs of critical points from $\mathcal{M}_{Y}(\mathfrak{s})$. Each is a smooth oriented manifold which can be compactfied to a smooth manifold with corners by adding broken flowlines that split through intermediate critical points.

The spectral flow of the Hessian operator of the Chern-Simons-Dirac functional defines a relative grading on $\mathcal{M}_{Y}(\mathfrak{s})$ :

$$
\operatorname{gr}(\cdot, \cdot): \quad \mathcal{M}_{Y}(\mathfrak{s}) \times \mathcal{M}_{Y}(\mathfrak{s}) \rightarrow \mathbb{Z} .
$$

In particular, using the unique reducible point $\theta$ in $\mathcal{M}_{Y}(\mathfrak{s})$, there is a $\mathbb{Z}$-lifting of the relative grading given by $\operatorname{gr}(a)=\operatorname{gr}(a, \theta)$.

Let $a$ be an irreducible monopole in $\mathcal{M}_{Y}(\mathfrak{s})$, then for any $b \neq a$ in $\mathcal{M}_{Y}(\mathfrak{s})$, the moduli space of flowlines from $a$ to $b$, denoted by $\mathcal{M}(a, b)$ has dimension $\operatorname{gr}(a)-\operatorname{gr}(b)>0$ (if non-empty). The moduli space of flowlines from $\theta$ to $d \in \mathcal{M}_{Y}^{*}(\mathfrak{s})$, denoted by $\mathcal{M}(\theta, d)$ has dimension $-g r(d)-1>0$ (if nonempty). Note that all these moduli spaces of flowlines admit an $\mathbb{R}$-action given by the $\mathbb{R}$-translation of flowlines: the corresponding quotient spaces are denoted by $\widehat{\mathcal{M}}(a, b)$ and $\widehat{\mathcal{M}}(\theta, d)$, respectively. 
For any irreducible critical points $a$ and $c$ in $\mathcal{M}_{Y}(\mathfrak{s})$ with $\operatorname{gr}(a)-\operatorname{gr}(c)=2$, we can construct a canonical complex line bundle over $\mathcal{M}(a, c)$ and a canonical section as follows (see section 5.3 in [5]). Choose a base point $\left(y_{0}, t_{0}\right)$ on $Y \times \mathbb{R}$, and a complex line $\ell_{y_{0}}$ in the fiber $W_{y_{0}}$ of the spinor bundle $W$ over $y_{0} \in Y$. We choose $\ell_{y_{0}}$ so that it does not contain the spinor part $\psi$ of any irreducible critical point. Since there are only finitely many critical points we can guarantee such choice exists. Denote the based moduli space of $\mathcal{M}(a, c)$ by $\mathcal{M}\left(O_{a}, O_{c}\right)$ as in [5], where $O_{a}$ and $O_{c}$ are the $U(1)$-orbits of monopoles on the based configuration space. We consider the line bundle

$$
\mathcal{L}_{a c}=\mathcal{M}\left(O_{a}, O_{c}\right) \times_{U(1)}\left(W_{y_{0}} / \ell_{y_{0}}\right) \rightarrow \mathcal{M}(a, c)
$$

with a section given by

$$
s([A, \Psi])=\left([A, \Psi], \Psi\left(y_{0}, t_{0}\right)\right) .
$$

For a generic choice of $\left(y_{0}, t_{0}\right)$ and $\ell_{y_{0}}$, the section $s$ of (8) has no zeroes on the boundary strata of the compactification of $\mathcal{M}(a, c)$. This determines a trivialization of $\mathcal{L}_{a c}$ away from a compact set in $\mathcal{M}(a, c)$. The line bundle $\mathcal{L}_{a c}$ over $\mathcal{M}(a, c)$, with the trivialization $\varphi$ specified above, has a well-defined relative Euler class (Cf. Lemma 5.7 in [5]).

Definition 2.1. $\quad$ 1. For any two irreducible critical points a and $b$ in $\mathcal{M}_{Y}(\mathfrak{s})$ with $\operatorname{gr}(a)-\operatorname{gr}(b)=1$, we define $n_{a b}:=\#(\hat{\mathcal{M}}(a, b))$, the number of flowlines in $\mathcal{M}(a, b)$ counting with orientations. Similarly, for any $a \in \mathcal{M}_{Y}(\mathfrak{s})$ with $\operatorname{gr}(a)=1$ and any $d \in \mathcal{M}_{Y}(\mathfrak{s})$ with $\operatorname{gr}(d)=-2$, we define $n_{a \theta}:=\#(\hat{\mathcal{M}}(a, \theta))$ and $n_{\theta d}:=\#(\hat{\mathcal{M}}(\theta, b))$, respectively.

2. For any two irreducible critical points a and c in $\mathcal{M}_{Y}(\mathfrak{s})$ with $\operatorname{gr}(a)-g r(c)=1$, we define $m_{a c}$ to be the relative Euler number of the canonical line bundle $\mathcal{L}_{a c}(7)$ with the canonical trivialization given by (8).

The following proposition states various relations satisfied by the integers defined in Definition 2.1, whose proof can be found in Remark 5.8 of [5].

Proposition 2.2. $\quad$ 1. For any irreducible critical point a in $\mathcal{M}_{Y}^{*}(\mathfrak{s})$ and any critical point c in $\mathcal{M}_{Y}(\mathfrak{s})$ with $\operatorname{gr}(a)-g r(c)=2$, we have the following identity:

$$
\sum_{\substack{b \in \mathcal{M}_{Y}^{*}(\mathfrak{s}) \\ g r(a)-g r(b)=1}} n_{a b} n_{b c}=0 .
$$


2. Let $a$ and $d$ be two irreducible critical points with $\operatorname{gr}(a)-g r(d)=3$. Assume that all the critical points $c$ with $\operatorname{gr}(a)>\operatorname{gr}(c)>\operatorname{gr}(d)$ are irreducible. Then we have the identity

$$
\sum_{c_{1}: g r(a)-g r\left(c_{1}\right)=1} n_{a, c_{1}} m_{c_{1}, d}-\sum_{c_{2}: g r\left(c_{2}\right)-g r(d)=1} m_{a, c_{2}} n_{c_{2}, d}=0 .
$$

When $\operatorname{gr}(a)=1$ and $\operatorname{gr}(d)=-2$, we have the identity

$$
\sum_{c_{1}: g r\left(c_{1}\right)=0} n_{a, c_{1}} m_{c_{1}, d}+n_{a \theta} n_{\theta d}-\sum_{c_{2}: g r\left(c_{2}\right)=-1} m_{a, c_{2}} n_{c_{2}, d}=0 .
$$

With the help of this Proposition, we can check that the equivariant Seiberg-Witten-Floer complex $C F_{*, U(1)}^{S W}(Y, \mathfrak{s})$ as given in (1) with the grading and the differential operator given by (2) and (3) is well-defined, and we denote its homology by $\operatorname{HF}_{*, U(1)}^{S W}(Y, \mathfrak{s})$. The equivariant Seiberg-Witten-Floer cohomology, denoted by $H F_{U(1)}^{S W, *}(Y, \mathfrak{s})$, is the homology of the dual complex $\operatorname{Hom}\left(C F_{*, U(1)}^{S W}(Y, \mathfrak{s}), \mathbb{Z}\right)$. The main result in [5] shows that the equivariant Seiberg-Witten Floer homology $H F_{*, U(1)}^{S W}(Y, \mathfrak{s})$ and cohomology $H F_{U(1)}^{S W, *}(Y, \mathfrak{s})$ are topological invariants of $(Y, \mathfrak{s})$.

\section{Variants of equivariant Seiberg-Witten Floer homology}

As mentioned in the introduction, we will generalize the construction of the equivariant Seiberg-Witten Floer homology in several ways.

First, we denote by $C F_{*, U(1)}^{S W, \infty}(Y, \mathfrak{s})$ the graded complex generated by

$$
\left\{\Omega^{k} \otimes \eta_{a}, \Omega^{k} \otimes 1_{a}, \Omega^{k} \otimes 1_{\theta}: a \in \mathcal{M}_{Y}^{*}(\mathfrak{s}), k \in \mathbb{Z} .\right\}
$$

More precisely, for any irreducible critical orbits $O_{a}$, we set

$$
\begin{aligned}
C_{*, U(1)}^{\infty}\left(O_{a}\right) & =\mathbb{Z}\left[\Omega, \Omega^{-1}\right] \otimes \Omega_{0}^{*}\left(O_{a}\right) \\
& :=\bigoplus_{k \in \mathbb{Z}}\left(\mathbb{Z} \Omega^{k} \otimes \eta_{a}+\mathbb{Z} \Omega^{k} \otimes 1_{a}\right)
\end{aligned}
$$

with the grading $\operatorname{gr}\left(\Omega^{k} \otimes \eta_{a}\right)=2 k+\operatorname{gr}(a)$ and $\operatorname{gr}\left(\Omega^{k} \otimes 1_{a}\right)=2 k+\operatorname{gr}(a)+1$, and we set

$$
C_{*, U(1)}^{\infty}(\theta)=\bigoplus_{k \in \mathbb{Z}} \mathbb{Z} \cdot \Omega^{k} \otimes 1_{\theta}
$$

with the grading $\operatorname{gr}\left(\Omega^{k} \otimes 1_{\theta}\right)=2 k$. 
We then consider

$$
C F_{*, U(1)}^{S W, \infty}(Y, \mathfrak{s})=\bigoplus_{a \in \mathcal{M}_{Y}(\mathfrak{s})} \mathbb{Z}\left[\Omega, \Omega^{-1}\right] \otimes \Omega_{0}^{*-\operatorname{dim}\left(O_{a}\right)}\left(O_{a}\right)
$$

with the grading and the differential operator given by $(2)$ and $(3)$ respectively. That is, $C F_{*, U(1)}^{S W, \infty}(Y, \mathfrak{s})$ is given by

$$
\begin{aligned}
& \bigoplus_{a \in \mathcal{M}_{Y}(\mathfrak{s})} C_{*, U(1)}^{\infty}\left(O_{a}\right) \\
= & \bigoplus_{a \in \mathcal{M}_{Y}^{*}(\mathfrak{s})} C_{*, U(1)}^{\infty}\left(O_{a}\right) \oplus C_{*, U(1)}^{\infty}(\theta) .
\end{aligned}
$$

Theorem 3.1. Define $H F_{*, U(1)}^{S W, \infty}(Y, \mathfrak{s})$ to be the homology of $\left(C F_{*, U(1)}^{S W, \infty}(Y, \mathfrak{s}), D\right)$. Then we have

$$
H F_{*, U(1)}^{S W, \infty}(Y, \mathfrak{s}) \cong \mathbb{Z}\left[\Omega, \Omega^{-1}\right]
$$

Proof. Consider the filtration of $C F_{*, U(1)}^{S W, \infty}(Y, \mathfrak{s})$ according to the grading of the critical points

$$
\mathcal{F}_{n} C_{*, U(1)}^{\infty}:=\bigoplus_{g r(a) \leq n} C_{*, U(1)}^{\infty}\left(O_{a}\right)
$$

the corresponding spectral sequence $E_{k l}^{r}$. The filtration is exhaustive, that is,

$$
C F_{*, U(1)}^{S W, \infty}(Y, \mathfrak{s})=\bigcup_{n} \mathcal{F}_{n} C_{*, U(1)}^{\infty}
$$

and

$$
\cdots \subset \mathcal{F}_{n-1} C_{*, U(1)}^{\infty} \subset \mathcal{F}_{n} C_{*, U(1)}^{\infty} \subset \mathcal{F}_{n+1} C_{*, U(1)}^{\infty} \subset \cdots \subset C F_{*, U(1)}^{S W, \infty}(Y, \mathfrak{s}) .
$$

Moreover, by the compactness of the moduli space of critical orbits, the set of indices $g r(a)$ is bounded from above and below, hence the filtration is bounded. Thus, the spectral sequence converges to $H F_{*, U(1)}^{S W, \infty}(Y, \mathfrak{s})$.

We compute the $E^{0}$-term:

$$
\begin{aligned}
E_{k l}^{0} & =\mathcal{F}_{k} C_{k+l, U(1)}^{\infty} / \mathcal{F}_{k-1} C_{k+l, U(1)}^{\infty} C_{k+l-i, U(1)}^{\infty}\left(O_{a}\right) / \bigoplus_{a \in \mathcal{M}_{Y}(\mathfrak{s}): \operatorname{gr}(a)=i \leq k-1} C_{k+l-i, U(1)}^{\infty}\left(O_{a}\right) \\
& =\bigoplus_{a \in \mathcal{M}_{Y}(\mathfrak{s}): \operatorname{gr}(a)=i \leq k} \bigoplus_{a \in \mathcal{M}_{Y}(\mathfrak{s}): \operatorname{gr}(a)=k} C_{l, U(1)}^{\infty}\left(O_{a}\right) .
\end{aligned}
$$


For $k \neq 0$ this complex is just the direct sum of the separate complexes $\left(C_{*, U(1)}^{\infty}\left(O_{a}\right), \partial_{U(1)}\right)$ on each orbit $O_{a}$ with $g r(a)=k$ :

$$
\cdots \rightarrow \mathbb{Z} . \Omega \otimes 1_{a} \stackrel{0}{\rightarrow} \mathbb{Z} . \Omega \otimes \eta_{a} \stackrel{-1}{\rightarrow} \mathbb{Z} .1 \otimes 1_{a} \stackrel{0}{\rightarrow} \mathbb{Z} .1 \otimes \eta_{a} \stackrel{-1}{\rightarrow} \mathbb{Z} . \Omega^{-1} \otimes 1_{a} \rightarrow \cdots
$$

In the case $k=0$ we have

$$
E_{0, l}^{0}=C_{l, U(1)}^{\infty}(\theta) \oplus \bigoplus_{a \in \mathcal{M}_{Y}^{*}(\mathfrak{s}): g r(a)=0} C_{l, U(1)}^{\infty}\left(O_{a}\right)
$$

which again is a direct sum of the complexes $\left(C_{*, U(1)}^{\infty}\left(O_{a}\right), \partial_{U(1)}\right)$, here $\partial_{U(1)}$ is the equivariant de Rham differential, and of the complex with generators $\left.\Omega^{r} \otimes 1_{\theta}\right\rangle$ in degree $l=2 r$ and trivial differentials.

We then compute the $E_{p q}^{1}$ term directly: we have

$$
E_{k l}^{1}=H_{k+l}\left(E_{k, *}^{0}\right)= \begin{cases}\mathbb{Z} . \Omega^{r} \otimes 1_{\theta} & k=0, l=2 r \\ 0 & k \neq 0,\end{cases}
$$

since each complex (10) is acyclic. Thus, the only non-trivial $E^{1}$-terms are of the form $E_{0 l}^{1}=\mathbb{Z} . \Omega^{r} \otimes 1_{\theta}$, $l=2 r$, with trivial differentials, so that the spectral sequence collapses and we obtain the result.

\subsection{Long exact sequence}

Definition 3.2. Let $C F_{*, U(1)}^{S W,-}(Y, \mathfrak{s})$ be the subcomplex of $C F_{*, U(1)}^{S W, \infty}(Y, \mathfrak{s})$, generated by

$$
\left\{\Omega^{k} \otimes \eta_{a}, \Omega^{k} \otimes 1_{a}, \Omega^{k} \otimes 1_{\theta}: a \in \mathcal{M}_{Y}^{*}(\mathfrak{s}), k \in \mathbb{Z} \text { and } k<0\right\},
$$

whose homology groups are denoted by $H F_{*, U(1)}^{S W,-}(Y, \mathfrak{s})$. The quotient complex is denoted by $C F_{*, U(1)}^{S W,+}(Y, \mathfrak{s})$, with the homology groups denoted by $H F_{*, U(1)}^{S W,+}(Y, \mathfrak{s})$.

Theorem 3.3. $\quad$ 1. $H F_{*, U(1)}^{S W,+}(Y, \mathfrak{s}) \cong H F_{*, U(1)}^{S W}(Y, \mathfrak{s})$, where $H F_{*, U(1)}^{S W}(Y, \mathfrak{s})$ is the equivariant SeibergWitten-Floer homology defined in [5].

2. There is an exact sequence of $\mathbb{Z}$-modules which relates these variants of equivariant Seiberg-WittenFloer homologies:

$$
\cdots \rightarrow H F_{*, U(1)}^{S W,-}(Y, \mathfrak{s}) \stackrel{l_{*}}{\longrightarrow} H F_{*, U(1)}^{S W, \infty}(Y, \mathfrak{s}) \stackrel{\pi_{*}}{\longrightarrow} H F_{*, U(1)}^{S W,+}(Y, \mathfrak{s}) \stackrel{\delta_{*}}{\longrightarrow} H F_{*-1, U(1)}^{S W,-}(Y, \mathfrak{s}) \rightarrow \cdots
$$


Proof. It is easy to see that $C F_{*, U(1)}^{S W,+}(Y, \mathfrak{s})=C F_{*, U(1)}^{S W}(Y, \mathfrak{s})$, with the same grading and differentials, hence $H F_{*, U(1)}^{S W,+}(Y, \mathfrak{s}) \cong H F_{* U(1)}^{S W}(Y, \mathfrak{s})$. The long exact sequence in homology is induced by the short exact sequence of chain complexes:

$$
0 \rightarrow C F_{*, U(1)}^{S W,-}(Y, \mathfrak{s}) \rightarrow C F_{*, U(1)}^{S W, \infty}(Y, \mathfrak{s}) \rightarrow C F_{*, U(1)}^{S W,+}(Y, \mathfrak{s}) \rightarrow 0
$$

From the above long exact sequence, we can define

$$
\begin{aligned}
H F_{\text {red,* }}^{S W}(Y, \mathfrak{s}) & =\operatorname{Coker}\left(\pi_{*}\right) \cong H F_{*, U(1)}^{S W,+}(Y, \mathfrak{s}) / \operatorname{Ker}\left(\delta_{*}\right) \\
& \cong \operatorname{Im}\left(\delta_{*}\right) \cong \operatorname{Ker}\left(l_{*-1}\right) .
\end{aligned}
$$

\subsection{The spectral sequence for $H F_{*, U(1)}^{S W,+}(Y, \mathfrak{s})$}

We consider again the filtration by index of critical orbits,

$$
\mathcal{F}_{n} C_{*, U(1)}^{+}:=\bigoplus_{\operatorname{gr}(a) \leq n} C_{*, U(1)}^{+}\left(O_{a}\right)
$$

for

$$
C_{*, U(1)}^{+}\left(O_{a}\right)=\mathbb{Z}[\Omega] \otimes \Omega_{0}^{*-\operatorname{dim}\left(O_{a}\right)}\left(O_{a}\right)
$$

We have

$$
\begin{aligned}
E_{k l}^{0} & =\mathcal{F}_{k} C_{k+l, U(1)}^{+} / \mathcal{F}_{k-1} C_{k+l, U(1)}^{+} \\
& =\bigoplus_{\operatorname{gr}(a)=k} C_{l, U(1)}^{+}\left(O_{a}\right) .
\end{aligned}
$$

This is a direct sum of the complexes

$$
\cdots \stackrel{-1}{\rightarrow} \mathbb{Z} . \Omega \otimes 1_{a} \stackrel{0}{\rightarrow} \mathbb{Z} . \Omega \otimes \eta_{a} \stackrel{-1}{\rightarrow} \mathbb{Z} .1 \otimes 1_{a} \stackrel{0}{\rightarrow} \mathbb{Z} .1 \otimes \eta_{a} \rightarrow 0
$$

over each orbit $O_{a} \cong S^{1}$ and, in the case $k=0$, the complex with generators $\Omega^{r} \otimes 1_{\theta}$ in degree $l=2 r$, and trivial differentials.

Thus, we obtain that $E_{p q}^{1}=H_{p+q}\left(E_{p *}^{0}\right)$ is of the form

$$
E_{p q}^{1}= \begin{cases}0 & q>0 \\ \mathbb{Z} .1 \otimes \eta_{a} & q=0, \operatorname{gr}(a)=p\end{cases}
$$


for $p \neq 0$, and

$$
E_{0 q}^{1}= \begin{cases}\mathbb{Z} . \Omega^{r} \otimes 1_{\theta} & q=2 r>0 \\ \mathbb{Z} .1 \otimes \eta_{a} \oplus \mathbb{Z} .1 \otimes 1_{\theta} & q=0, \operatorname{gr}(a)=0 .\end{cases}
$$

The differential $d^{1}: E_{p, q}^{1} \rightarrow E_{p-1, q}^{1}$ is of the form

$$
\begin{aligned}
d^{1}\left(1 \otimes \eta_{a}\right)= & n_{a b} 1 \otimes \eta_{b} \\
& +n_{a \theta} 1 \otimes 1_{\theta} \quad(\text { if } \operatorname{gr}(a)=1)
\end{aligned}
$$

Thus, we obtain

$$
E_{p q}^{2}= \begin{cases}H F_{p}^{S W}(Y, \mathfrak{s}) & p \neq 0, q=0 \\ \operatorname{Ker}\left(\Delta_{1}\right) & p=1, q=0 \\ H F_{0}^{S W}(Y, \mathfrak{s}) \oplus T_{0} & p=0, q=0 \\ \mathbb{Z} . \Omega^{r} \otimes 1_{\theta} & p=0, q=2 r>0 .\end{cases}
$$

Here $H F_{*}^{S W}(Y, \mathfrak{s})$ denotes the non-equivariant (metric and perturbation dependent) Seiberg-Witten Floer homology. This is the homology of the complex with generators $1 \otimes \eta_{a}$ in degree $\operatorname{gr}(a)$ and boundary coefficients $n_{a b}$ for $\operatorname{gr}(a)-\operatorname{gr}(b)=1$. We also denoted by $\Delta_{1}$ the map

$$
\begin{gathered}
\Delta_{1}: H_{1}^{S W}(Y, \mathfrak{s}) \rightarrow \mathbb{Z} .1 \otimes 1_{\theta}, \\
\Delta_{1}\left(\sum x_{a} 1 \otimes \eta_{a}\right)=\sum x_{a} n_{a \theta} 1 \otimes 1_{\theta},
\end{gathered}
$$

where the coefficients $x_{a}$ satisfy $\sum x_{a} n_{a b}=0$. Finally, the term $T_{0}$ denotes the term

$$
T_{0}=\mathbb{Z} .1 \otimes 1_{\theta} / \operatorname{Im}\left(\Delta_{1}\right)
$$

Notice then that the boundary $d^{2}: E_{p, q}^{2} \rightarrow E_{p-2, q+1}^{2}$ is trivial, hence the $E_{p, q}^{3}$ terms are disposed as in the diagram:

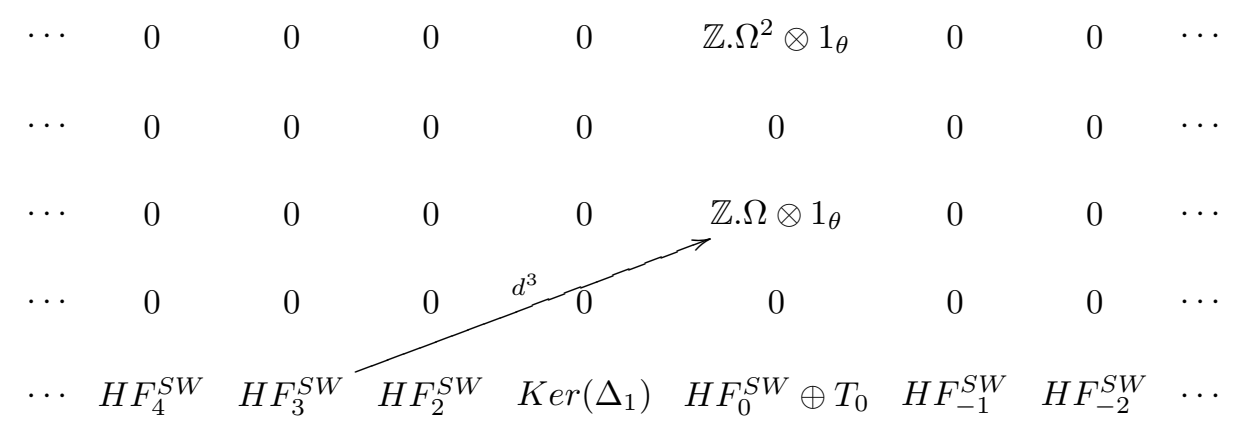


The differential $d^{3}: E_{p, q}^{3} \rightarrow E_{p-3, q+2}^{3}$ is given by the expression

$$
d^{3}\left(\left[\sum x_{a} 1 \otimes \eta_{a}\right]\right)=\sum x_{a} m_{a c} n_{c \theta} \Omega \otimes 1_{\theta},
$$

for $\operatorname{gr}(a)-\operatorname{gr}(c)=2$. The expression is obtained by considering the unique choice of a representative of the class $\left[\sum x_{a} 1 \otimes \eta_{a}\right]$ in $E_{p, q}^{3}$ whose boundary (3) defines a class in $E_{p-3, q+2}^{3}$.

The differential $d^{4}: E_{p, q}^{4} \rightarrow E_{p-4, q+3}^{4}$ is again trivial, and we obtain the $E_{p q}^{5}$ of the form

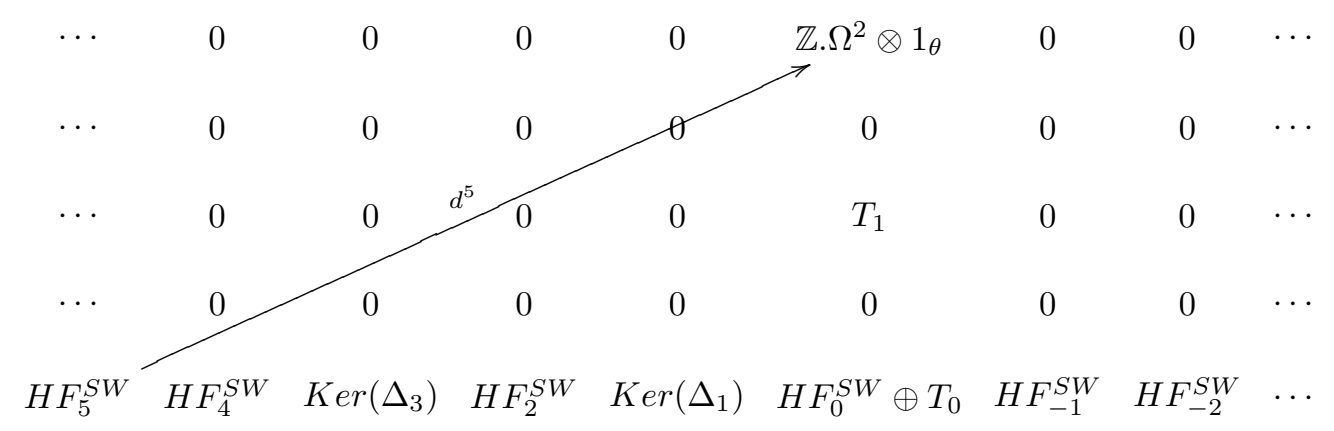

where again we denote by $T_{1}$ the term

$$
T_{1}=\mathbb{Z} \cdot \Omega \otimes 1_{\theta} / \operatorname{Im}\left(\Delta_{3}\right)
$$

Thus, by iterating the process, we observe that all the differentials $d^{2 k}: E_{p, q}^{2 k} \rightarrow E_{p-2 k, q+2 k+1}^{2 k}$ are trivial and the differentials $d^{2 k+1}: E_{p, q}^{2 k+1} \rightarrow E_{p-2 k-1, q+2 k}^{2 k+1}$ consists of one map for $p=2 k+1, q=0$ :

$$
\Delta_{2 k+1}: H F_{2 k+1}^{S W} \rightarrow \mathbb{Z} . \Omega^{k} \otimes 1_{\theta}
$$

induced by

$$
\Delta_{2 k+1}\left(\sum x_{a} 1 \otimes \eta_{a}\right)=\sum x_{a} m_{a a_{2 k-1}} m_{a_{2 k-1} a_{2 k-3}} \cdots m_{a_{3} a_{1} n_{a_{1} \theta} \Omega^{k} \otimes 1_{\theta} .}
$$

Here we have $\operatorname{gr}(a)=2 k+1$ and $\operatorname{gr}\left(a_{r}\right)=r$. Notice that these maps agree with the morphism $\Delta_{*}$, which is obtained in [5] as the connecting homomorphism in the long exact sequence relating equivariant and non-equivariant Seiberg-Witten Floer homologies.

We thus obtain the following structure theorem for equivariant Seiberg-Witten Floer homology. 
Theorem 3.4. The equivariant Seiberg-Witten Floer homology $H F_{*, U(1)}^{S W,+}(Y, \mathfrak{s})$ has the form

$$
H F_{*, U(1)}^{S W,+}(Y, \mathfrak{s})= \begin{cases}\operatorname{Ker}\left(\Delta_{2 k+1}\right) & *=2 k+1>0 \\ \operatorname{HF}_{2 k}^{S W}(Y, \mathfrak{s}) \oplus T_{k} & *=2 k \geq 0 \\ H F_{*}^{S W}(Y, \mathfrak{s}) & *<0\end{cases}
$$

where $T_{k}$ is the term

$$
T_{k}=\mathbb{Z} \cdot \Omega^{k} \otimes 1_{\theta} / \operatorname{Im}\left(\Delta_{2 k+1}\right)
$$

This result refines the long exact sequence obtained in [5]:

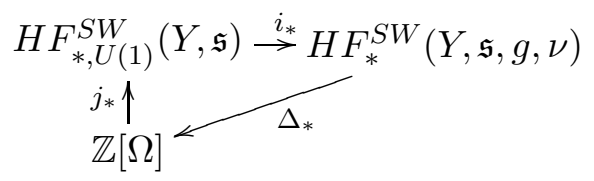

Similar results can be obtained for $H F_{*, U(1)}^{S W,-}(Y, \mathfrak{s})$.

\subsection{Topological invariance}

Note that the definitions of these homologies depend on the Seiberg-Witten equations, which use the metric and perturbation on $(Y, \mathfrak{s})$. By the result of [5], we know that $H F_{*, U(1)}^{S W,+}(Y, \mathfrak{s}) \cong H F_{*, U(1)}^{S W}(Y, \mathfrak{s})$ is a topological invariant of $(Y, \mathfrak{s})$, we first recall this topological invariance as stated in Theorem 6.1 [5].

Theorem 3.5. (Theorem 6.1 [5]) Let $(Y, \mathfrak{s})$ be a rational homology sphere with a Spin ${ }^{c}$ structure. Suppose given two metrics $g_{0}$ and $g_{1}$ on $Y$ and perturbations $\nu_{0}$ and $\nu_{1}$ such that $\operatorname{Ker}\left(\not_{\nu_{0}}^{g_{0}}\right)=\operatorname{Ker}\left(\not \not_{\nu_{1}}^{g_{1}}\right)=0$, so that the corresponding monopole moduli spaces $\mathcal{M}_{Y}\left(\mathfrak{s}, g_{0}, \nu_{0}\right)$ and $\mathcal{M}_{Y}\left(\mathfrak{s}, g_{1}, \nu_{1}\right)$ consist of finitely many isolated points. Then there exists an isomorphism between the equivariant Seiberg-Witten Floer homologies $H F_{*, U(1)}^{S W}\left(Y, \mathfrak{s}, g_{0}, \nu_{0}\right)$ and $H F_{*, U(1)}^{S W}\left(Y, \mathfrak{s}, g_{1}, \nu_{1}\right)$, with a degree shift given by the spectral flow of the Dirac operator $\partial_{\nu_{t}}^{g_{t}}$ along a path of metrics and perturbations connecting $\left(g_{0}, \nu_{0}\right)$ and $\left(g_{1}, \nu_{1}\right)$. That is, if the complex spectral flow along the path $\left(g_{t}, \nu_{t}\right)$ is denoted by $S F_{\mathbb{C}}\left(\not \partial_{\nu_{t}}^{g_{t}}\right)$, then for any $k \in \mathbb{Z}$,

$$
H F_{k, U(1)}^{S W}\left(Y, \mathfrak{s}, g_{0}, \nu_{0}\right) \cong H F_{k+2 S F_{\mathbb{C}}\left(\not_{\nu_{t}}^{g_{t}}\right), U(1)}^{S W}\left(Y, \mathfrak{s}, g_{1}, \nu_{1}\right)
$$

From Theorem 3.1, we know that

$$
H F_{*, U(1)}^{S W, \infty}(Y, \mathfrak{s}) \cong \mathbb{Z}\left[\Omega, \Omega^{-1}\right]
$$


is independent of $(Y, \mathfrak{s})$, up to a degree shift as given in Theorem 3.5. Thus, applying the five lemma to the long exact sequence in Theorem 3.3, we obtain that $H F_{*, U(1)}^{S W,-}(Y, \mathfrak{s})$ and $H F_{r e d, *}^{S W}(Y, \mathfrak{s})$ are also topological invariants of $(Y, \mathfrak{s})$.

Theorem 3.6. $H F_{*, U(1)}^{S W,-}(Y, \mathfrak{s})$ and $H F_{r e d, *}^{S W}(Y, \mathfrak{s})$ are topological invariants of $(Y, \mathfrak{s})$, in the sense that, given any two metrics $g_{0}$ and $g_{1}$ on $Y$ and perturbations $\nu_{0}$ and $\nu_{1}$, with $\operatorname{Ker}\left(\not_{\nu_{0}}^{g_{0}}\right)=\operatorname{Ker}\left(\not \partial_{\nu_{1}}^{g_{1}}\right)=0$, there exist isomorphisms

$$
\begin{aligned}
& H F_{k, U(1)}^{S W,-}\left(Y, \mathfrak{s}, g_{0}, \nu_{0}\right) \cong H F_{k+2 S F_{\mathbb{C}}\left(\partial_{\nu_{t}}^{g t}\right), U(1)}^{S W,-}\left(Y, \mathfrak{s}, g_{1}, \nu_{1}\right) \\
& H F_{r e d, k}^{S W}\left(Y, \mathfrak{s}, g_{0}, \nu_{0}\right) \cong H F_{r e d, k+2 S F_{\mathbb{C}}\left(\not_{\nu_{t}}^{g_{t}}\right)}^{S W}\left(Y, \mathfrak{s}, g_{1}, \nu_{1}\right) .
\end{aligned}
$$

Here $S F_{\mathbb{C}}\left(\not_{\nu_{t}}^{g_{t}}\right)$ denotes the complex spectral flow of the Dirac operator $\not_{\nu_{t}}^{g_{t}}$ along the path $\left(g_{t}, \nu_{t}\right)$.

\section{Properties of equivariant Seiberg-Witten Floer homologies}

In this section, we briefly discuss some of the algebraic structures and properties of the equivariant Seiberg-Witten Floer homologies defined in the previous section.

Note that for any irreducible critical points $a$ and $b$ in $\mathcal{M}_{Y}^{*}(\mathfrak{s})$, the associated integer $m_{a c}$ is the counting of points in the geometric representative of the relative first Chern class of the canonical line bundle (7) over $\mathcal{M}(a, c)$, we can apply this fact to define a $u$-action on the chain complex $C F_{*, U(1)}^{S W, \infty}(Y, \mathfrak{s})$

$$
u: \quad C F_{*, U(1)}^{S W, \infty}(Y, \mathfrak{s}) \longrightarrow C F_{*, U(1)}^{S W, \infty}(Y, \mathfrak{s})
$$

which decreases the grading by two. The action is given in terms of its actions on generators as follows:

$$
\begin{aligned}
u\left(\Omega^{n} \otimes \eta_{a}\right) & =\sum_{\substack{c \in \mathcal{M}^{*}(Y, \mathfrak{s}) \\
g r(a)-g r(c)=2}} m_{a c} \Omega^{n} \otimes \eta_{c} . \\
u\left(\Omega^{n} \otimes 1_{a}\right) & = \begin{cases}\sum_{\substack{c \in \mathcal{M}^{*}(Y, \mathfrak{s}) \\
g r(a)-g r(c)=2}} m_{a c} \Omega^{n} \otimes 1_{c} & \text { if } g r(a) \neq 1 \\
\sum_{\substack{c \in \mathcal{M}^{*}(Y, \mathfrak{s}) \\
g r(c)=-1}} m_{a c} \Omega^{n} \otimes 1_{c}+n_{a \theta} \Omega^{n} \otimes 1_{\theta} & \text { if } g r(a)=1\end{cases} \\
u\left(\Omega^{n} \otimes 1_{\theta}\right) & =\sum_{\substack{d \in \mathcal{M}_{Y}^{*}(\mathfrak{s}) \\
g r(d)=-2}} n_{\theta d} \Omega^{n} \times \eta_{d}+\Omega^{n-1} \otimes 1_{\theta} .
\end{aligned}
$$


Proposition 4.1. The u-action defined (14) on the chain complex $C F_{*, U(1)}^{S W, \infty}(Y, \mathfrak{s})$ is homotopic to the $\Omega^{-1}$-action acting on $C F_{*, U(1)}^{S W, \infty}(Y, \mathfrak{s})$. The induced actions on $C F_{*, U(1)}^{S W, \pm}(Y, \mathfrak{s})$ define $\mathbb{Z}[u]$-module structures on $H F_{*, U(1)}^{S W, \pm}(Y, \mathfrak{s})$.

Proof. Define $H: C F_{*, U(1)}^{S W, \infty}(Y, \mathfrak{s}) \longrightarrow C F_{*, U(1)}^{S W, \infty}(Y, \mathfrak{s})$ by its actions on the generators as follows:

$$
\begin{aligned}
& H\left(\Omega^{n} \otimes \eta_{a}\right)=0, \\
& H\left(\Omega^{n} \otimes 1_{a}\right)=\Omega^{n} \otimes \eta_{a}, \\
& H\left(\Omega^{n} \otimes 1_{\theta}\right)=0 .
\end{aligned}
$$

Then it is a direct calculation to show that we have:

$$
\begin{gathered}
\left(u-\Omega^{-1}\right)\left(\Omega^{k} \otimes \eta_{a}\right)=m_{a c} \Omega^{k} \otimes \eta_{c}-\Omega^{k-1} \otimes \eta_{a}=(D H+H D)\left(\Omega^{k} \otimes \eta_{a}\right) \\
\left(u-\Omega^{-1}\right)\left(\Omega^{k} \otimes 1_{a}\right)=m_{a c} \Omega^{k} \otimes 1_{c}-\Omega^{n-1} \otimes 1_{a}\left(+n_{a \theta} \Omega^{n} \otimes 1_{\theta} \text { if } \operatorname{gr}(a)=1\right)=(D H+H D)\left(\Omega^{k} \otimes 1_{a}\right), \\
\left(u-\Omega^{-1}\right)\left(\Omega^{k} \otimes 1_{\theta}\right)=n_{\theta d} \Omega^{n} \otimes \eta_{d}=(D H+H D)\left(\Omega^{k} \otimes 1_{\theta}\right) .
\end{gathered}
$$

Thus the claim follows using the chain homotopy $u-\Omega^{-1}=D \circ H+H \circ D$.

Thus, on the homological level, we can identify the $u$-action with the induced $\Omega^{-1}$ action on various homologies. In particular, we see that there is a subcomplex $\widehat{C F}_{*}^{S W}(Y, \mathfrak{s})$ of $C F_{*, U(1)}^{S W,+}(Y, \mathfrak{s})$ such that the following short exact sequence of chain complexes holds:

$$
0 \rightarrow \widehat{C F}_{*}^{S W}(Y, \mathfrak{s}) \longrightarrow C F_{*, U(1)}^{S W,+}(Y, \mathfrak{s}) \stackrel{\Omega^{-1}}{\longrightarrow} C F_{*, U(1)}^{S W,+}(Y, \mathfrak{s}) \rightarrow 0
$$

Proposition 4.2. Let $\widehat{H F}_{*}^{S W}(Y, \mathfrak{s})$ be the homology of $\widehat{C F}_{*}^{S W}(Y, \mathfrak{s})$, then $\widehat{H F}_{*}^{S W}(Y, \mathfrak{s})$ is also a topological invariant of $(Y, \mathfrak{s})$, and it is determined by the following long exact sequence

$$
\cdots \rightarrow \widehat{H F}_{*}^{S W}(Y, \mathfrak{s}) \longrightarrow H F_{*, U(1)}^{S W,+}(Y, \mathfrak{s}) \stackrel{u}{\longrightarrow} H F_{*-2, U(1)}^{S W,+}(Y, \mathfrak{s}) \longrightarrow \widehat{H F}_{*-1}^{S W}(Y, \mathfrak{s}) \rightarrow \cdots
$$

Moreover, $\widehat{H F}^{S W}(Y, \mathfrak{s})$ is non-trivial if and only if $H_{*, U(1)}^{S W,+}(Y, \mathfrak{s})$ is non-trivial.

Proof. The long exact sequence follows from the short exact sequence of chain complexes (15) and Proposition 4.1. This long exact sequence implies that $\widehat{H F}_{*}^{S W}(Y, \mathfrak{s})$ is also a topological invariant of $(Y, \mathfrak{s})$. 
Note that, from the compactness of $\mathcal{M}_{Y}(\mathfrak{s})$, we see that each element in $H F_{*, U(1)}^{S W,+}(Y, \mathfrak{s})$ can be annihilated by a sufficiently large power of $\Omega^{-1}$. Hence, $u$ is an isomorphism on $H F_{*, U(1)}^{S W,+}(Y, \mathfrak{s})$ if and only if $H F_{*, U(1)}^{S W,+}(Y, \mathfrak{s})$ is trivial. Then the last claim follows from this observation and the long exact sequence.

If we think of the set of $\operatorname{Spin}^{c}$ structures on $Y$ as the set of equivalence classes of nowhere vanishing vector fields on $Y$ (Cf.[9]), then there is a natural bijection between $\operatorname{Spin}^{c}(Y)$ and $\operatorname{Spin}^{c}(-Y)$ where $-Y$ is the same $Y$ with the opposite orientation.

Theorem 4.3. Let $(Y, \mathfrak{s})$ be a rational homology 3-sphere with a $\operatorname{Spin}^{c}$ structure $\mathfrak{s}$, and $(-Y, \mathfrak{s})$ denote $Y$ with the opposite orientation and the corresponding $\operatorname{Spin}^{c}$ structure. Then there is a natural isomorphism

$$
H F_{U(1)}^{S W, *}(Y, \mathfrak{s}) \cong H F_{*, U(1)}^{S W,-}(-Y, \mathfrak{s})
$$

where $H_{U(1)}^{S W, *}(Y, \mathfrak{s})$ is the equivariant Seiberg-Witten-Floer cohomology defined in [5].

Proof. Note that $H F_{U(1)}^{S W, *}(Y, \mathfrak{s})$ is the homology of the dual complex $\operatorname{Hom}\left(C F_{*, U(1)}^{S W,+}(Y, \mathfrak{s}), \mathbb{Z}\right)$. We start to construct a natural pairing

$$
\langle\cdot, \cdot\rangle: \quad C F_{*, U(1)}^{S W, \infty}(Y, \mathfrak{s}) \times C F_{*, U(1)}^{S W, \infty}(-Y, \mathfrak{s}) \longrightarrow \mathbb{Z}
$$

which satisfies

$$
\left\langle D_{Y}\left(\xi_{1}\right), \xi_{2}\right\rangle=\left\langle\xi_{1}, D_{-Y}\left(\xi_{2}\right)\right\rangle, \quad\left\langle\Omega^{-1}\left(\xi_{1}\right), \xi_{2}\right\rangle=\left\langle\xi_{1}, \Omega^{-1}\left(\xi_{2}\right)\right\rangle
$$

for any element $\xi_{1} \in C F_{*, U(1)}^{S W, \infty}(Y, \mathfrak{s})$ and any element $\xi_{2} \in C F_{*, U(1)}^{S W, \infty}(-Y, \mathfrak{s})$.

Then we will show that the above pairing is non-degenerate when restricted to $C F_{*, U(1)}^{S W,+}(Y, \mathfrak{s}) \times C F_{*, U(1)}^{S W,--}(-Y, \mathfrak{s})$.

From the nature of the Seiberg-Witten equations, we see that there is an identification

$$
\mathcal{M}_{Y}(\mathfrak{s}) \rightarrow \mathcal{M}_{-Y}(\mathfrak{s})
$$

for a good pair of metric and perturbation on $(Y, \mathfrak{s})$ and the corresponding metric and perturbation on $(-Y, \mathfrak{s})$. Then the relative gradings with respect to the unique reducible monopole in $\mathcal{M}_{Y}(\mathfrak{s})$ and $\mathcal{M}_{-Y}(\mathfrak{s})$ respectively, satisfies

$$
g r_{-Y}\left(a^{-}\right)=-g r_{Y}(a)-1
$$


where $a^{-}$is the element in $\mathcal{M}_{-Y}^{*}(\mathfrak{s})$ corresponding to $a \in \mathcal{M}_{Y}^{*}(\mathfrak{s})$, we assume that $\operatorname{gr}_{Y}(\theta)=g r_{-Y}\left(\theta^{-}\right)$. Moreover, there is an natural identification between the moduli spaces of flowlines for $(Y, \mathfrak{s})$ and $(-Y, \mathfrak{s})$, that is,

$$
\mathcal{M}_{Y \times \mathbb{R}}(a, b) \cong \mathcal{M}_{-Y \times \mathbb{R}}\left(b^{-}, a^{-}\right) .
$$

Now we define the pairing on $C F_{*, U(1)}^{S W, \infty}(Y, \mathfrak{s}) \times C F_{*, U(1)}^{S W, \infty}(-Y, \mathfrak{s})$ such that the following pairings are the only non-trivial pairings:

$$
\begin{gathered}
\left\langle\Omega^{n} \otimes \eta_{a}, \Omega^{-n-1} \otimes 1_{a^{-}}\right\rangle=1 \\
\left\langle\Omega^{n} \otimes 1_{a}, \Omega^{-n-1} \otimes \eta_{a^{-}}\right\rangle=1 \\
\left\langle\Omega^{n} \otimes 1_{\theta}, \Omega^{-n-1} \otimes 1_{\theta^{-}}\right\rangle=1 .
\end{gathered}
$$

It is a direct calculation to show that this pairing satisfies the relation (17) and the restriction of this pairing to $C F_{*, U(1)}^{S W,+}(Y, \mathfrak{s}) \times C F_{*, U(1)}^{S W,-}(-Y, \mathfrak{s})$ is non-degenerate. Then the claim follows from the definition.

Let $\widehat{H F}^{S W, *}(Y, \mathfrak{s})$ and $H F_{ \pm, U(1)}^{S W, *}(Y, \mathfrak{s})$ denote the homology groups of the dual complexes $\operatorname{Hom}\left(\widehat{C F}_{*}^{S W}(Y, \mathfrak{s}), \mathbb{Z}\right)$ and $\operatorname{Hom}\left(C F_{*, U(1)}^{S W, \pm}(Y, \mathfrak{s}), \mathbb{Z}\right)$ of $\widehat{C F}_{*}^{S W}(Y, \mathfrak{s})$ and $C F_{*, U(1)}^{S W, \pm}(Y, \mathfrak{s})$ respectively. From the proof the above Theorem 4.3, we actually establish the following duality between these homologies.

Theorem 4.4. For any rational homology 3-sphere $Y$ with a spinc structure $\mathfrak{s}$, there exist natural isomorphisms

$$
\widehat{H F}^{S W, *}(Y, \mathfrak{s}) \cong \widehat{H F}_{*}^{S W}(-Y, \mathfrak{s}), \quad H F_{ \pm, U(1)}^{S W, *}(Y, \mathfrak{s}) \cong H F_{*, U(1)}^{S W, \mp}(-Y, \mathfrak{s})
$$

\section{References}

[1] D.M. Austin, P.J. Braam, Equivariant Floer theory and gluing Donaldson polynomials, Topology 35 (1996), No.1, 167-200.

[2] A.L. Carey, B.L. Wang, Seiberg-Witten-Floer homology and Gluing formulae, preprint.

[3] K.A. Froyshov, The Seiberg-Witten equations and four-manifolds with boundary, Math. Res. Lett. 3 (1996), N.3, 373-390. 
[4] M. Marcolli, Seiberg-Witten gauge theory, Texts and Readings in Mathematics, 17. Hindustan Book Agency, New Delhi, 1999.

[5] M.Marcolli and B.L.Wang, Equivariant Seiberg-Witten Floer homology, Commun. Anal. Geom. Vol.9 N.3 (2001) 451-639.

[6] M. Marcolli, B.L. Wang, Seiberg-Witten invariant and Casson-Walker invariant for any rational homology 3-sphere, Geometriae Dedicata. Vol.91 (1): 45-58, April 2002.

[7] P. Ozsvath, Z. Szabo, Holomorphic disks and topological invariants for closed three-manifolds, math.SG/0101206.

[8] P. Ozsvath, Z. Szabo, Holomorphic disks and three-manifold invariants: properties and applications, math.SG/0105202.

[9] V. Turaev, Torsion invariants of $\operatorname{Spin}^{c}$ structures on 3-manifolds, Math. Research Letters, 4, 679$695,1997$.

\section{Matilde Marcolli and Bai-Ling Wang,}

Max-Planck-Institut für Mathematik, Vivatsgasse 7, D-53111 Bonn, Germany.

marcolli@mpim-bonn.mpg.de

bwang@mpim-bonn.mpg.de 